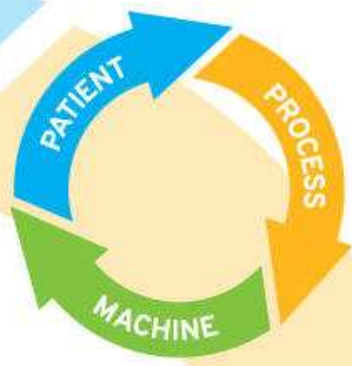

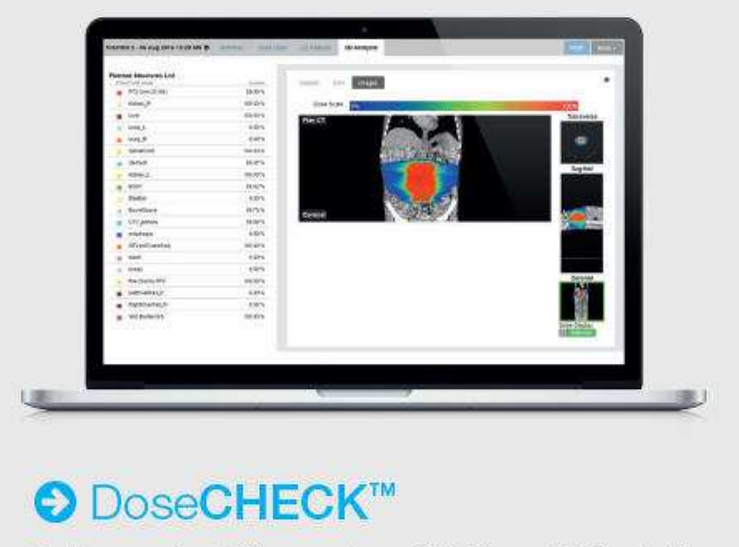

Independent Secondary 3D Dose Calculations

Generate a full 3D dose volume to compare against a TPS-generated 3D dose volume, for verification of complex treatment fields.

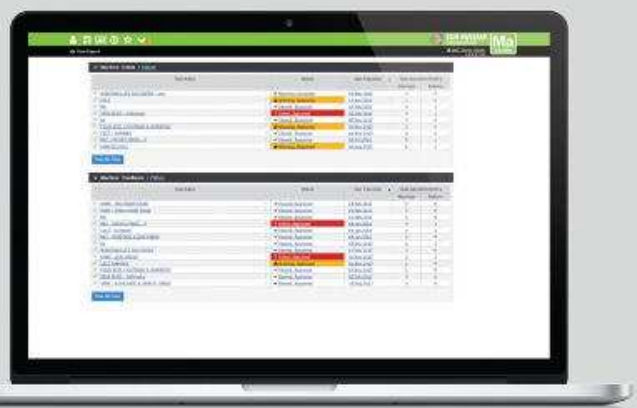

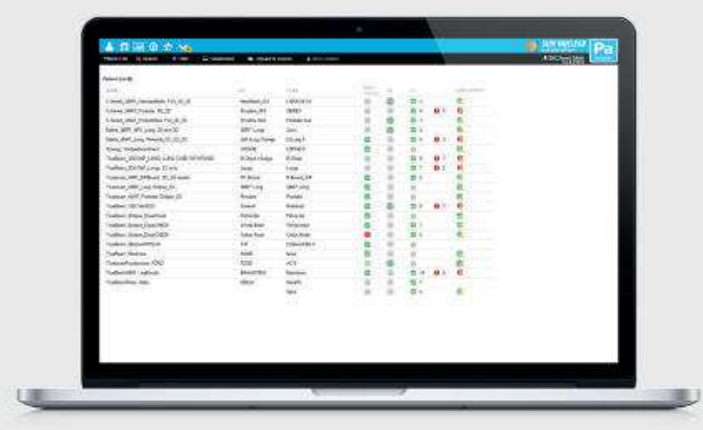

\section{PerFRACTION ${ }^{\mathrm{TM}}$}

\section{Fraction $0^{\text {tm }}-3 D$ Pre-Treatment QA}

Perform fully-automated phantom-less, EPID-based

QA for independent measurement and analysis of 3D dose.

Fraction $\mathbf{n}^{\text {TM }}-3 D$ In-Vivo Monitoring Accurately and efficiently identify, isolate and understand the impact of set-up errors and anatomy changes on $3 \mathrm{D}$ dose deposited in the patient. 


\title{
Improved image quality in pinhole SPECT by accurate modeling of the point spread function in low magnification systems
}

\author{
Francisco Pino \\ Unitat de Biofísica, Facultat de Medicina, Universitat de Barcelona, Barcelona 08036, Spain and Servei de \\ Física Mèdica i Protecció Radiològica, Institut Català d'Oncologia, L'Hospitalet de Llobregat 08907, Spain
}

Nuria Roé

Unitat de Biofísica, Facultat de Medicina, Universitat de Barcelona, Barcelona 08036, Spain

Pablo Aguiara)

Fundación Ramón Domínguez, Complexo Hospitalario Universitario de Santiago de Compostela 15706, Spain and Grupo de Imagen Molecular, Instituto de Investigacións Sanitarias de Santiago de Compostela (IDIS), Galicia 15782, Spain

Carles Falcon and Domènec Ros

Institut d'Investigacions Biomèdiques August Pi i Sunyer (IDIBAPS), Barcelona 08036, Spain and CIBER en Bioingeniería, Biomateriales y Nanomedicina (CIBER-BBN), Barcelona 08036, Spain

\author{
Javier Pavía \\ Institut d'Investigacions Biomèdiques August Pi i Sunyer (IDIBAPS), Barcelona 080836, Spain; CIBER en \\ Bioingeniería, Biomateriales y Nanomedicina (CIBER-BBN), Barcelona 08036, Spain; and Servei de Medicina \\ Nuclear, Hospital Clínic, Barcelona 08036, Spain
}

(Received 24 February 2014; revised 10 October 2014; accepted for publication 7 December 2014; published 14 January 2015)

Purpose: Single photon emission computed tomography (SPECT) has become an important noninvasive imaging technique in small-animal research. Due to the high resolution required in small-animal SPECT systems, the spatially variant system response needs to be included in the reconstruction algorithm. Accurate modeling of the system response should result in a major improvement in the quality of reconstructed images. The aim of this study was to quantitatively assess the impact that an accurate modeling of spatially variant collimator/detector response has on image-quality parameters, using a low magnification SPECT system equipped with a pinhole collimator and a small gamma camera.

Methods: Three methods were used to model the point spread function (PSF). For the first, only the geometrical pinhole aperture was included in the PSF. For the second, the septal penetration through the pinhole collimator was added. In the third method, the measured intrinsic detector response was incorporated. Tomographic spatial resolution was evaluated and contrast, recovery coefficients, contrast-to-noise ratio, and noise were quantified using a custom-built NEMA NU 4-2008 image-quality phantom.

Results: A high correlation was found between the experimental data corresponding to intrinsic detector response and the fitted values obtained by means of an asymmetric Gaussian distribution. For all PSF models, resolution improved as the distance from the point source to the center of the field of view increased and when the acquisition radius diminished. An improvement of resolution was observed after a minimum of five iterations when the PSF modeling included more corrections. Contrast, recovery coefficients, and contrast-to-noise ratio were better for the same level of noise in the image when more accurate models were included. Ring-type artifacts were observed when the number of iterations exceeded 12.

Conclusions: Accurate modeling of the PSF improves resolution, contrast, and recovery coefficients in the reconstructed images. To avoid the appearance of ring-type artifacts, the number of iterations should be limited. In low magnification systems, the intrinsic detector PSF plays a major role in improvement of the image-quality parameters. (c) 2015 American Association of Physicists in Medicine. [http://dx.doi.org/10.1118/1.4905157]

Key words: pinhole, modeling PSF, image quality, SPECT, quantification, small animal

\section{INTRODUCTION}

Single photon emission computed tomography (SPECT) has become an important noninvasive imaging technique in small-animal research, ${ }^{1,2}$ allowing the in vivo measurement of concentrations of radiolabeled biomolecules in the various organs and tissues of laboratory animals. Ideally, the reconstructed image should reflect the true activity concentration. Much of the work on small-animal SPECT has focused on spatial resolution improvement using pinhole 
collimation, and a variety of strategies have been adopted in the development of new equipment. One of these uses clinical gamma cameras equipped with multiple pinhole collimators, which, due to their high magnification and high sensitivity, allows a high resolution to be achieved. ${ }^{3-6}$ Another involves the use of small cameras. ${ }^{7-10}$ These are low-cost systems, and due to their flexibility and size, they are suitable for molecular imaging multimodality. To compensate for the lower magnification inherent to small cameras, this system should allow the radius of rotation (ROR) to be fitted to the characteristics of the study, thereby improving resolution and sensitivity.

Most of the scientific literature on image quality in smallanimal SPECT is based on a qualitative evaluation of the effective spatial resolution. ${ }^{3,5-9}$ Recently, some authors have focused their attention on the quantification of system resolution as well as other quantitative parameters related to image quality, such as absolute quantification, ${ }^{11-13}$ recovery coefficients (RC), and noise. ${ }^{12,14}$ These studies characterize the image-quality parameters as a function of reconstruction settings and of the correction of attenuation and scatter in the object. The effect of the reconstruction algorithms on quantification has also been studied. Previous studies have assessed the impact that accurate point spread function (PSF) modeling can have on image resolution. ${ }^{15,16}$ Vanhove et al. ${ }^{15}$ showed that modeling the pinhole aperture led to a better trade-off between spatial resolution and noise in reconstructed images. Feng et al. ${ }^{16}$ concluded that the modeling of the PSF including septal penetration increases resolution in reconstructed images for single-pinhole and multipinhole imaging. Although interesting, these studies were performed using high magnification equipment in which the intrinsic detector response is of less importance. For low magnification equipment, modeling intrinsic detector response would be expected to have a greater effect on resolution and also the quantification of other image-quality parameters. To the best of our knowledge, the effect of PSF modeling, including the intrinsic detector response, for low magnification scanners has not yet been studied in depth.

The aim of this study was to assess how an accurate modeling of the system PSF impacts on spatial resolution, contrast, recovery coefficients, contrast-to-noise ratio (CNR), and noise in reconstructed images, using a low magnification SPECT system consisting of a small gamma camera equipped with a pinhole collimator. Three methods were used to model the PSF. For the first, only the geometrical pinhole aperture was included in the PSF. In the second, septal penetration throughout the pinhole collimator was added, using a previously proposed attenuation model. ${ }^{17,18}$ In the third method, the intrinsic detector response was measured and incorporated.

\section{METHODS}

\section{A. Reconstruction algorithm}

A reconstruction method based on the ordered subsets expectation maximization (3D-OSEM) algorithm was implemented. The elements $a_{i j}$ of the system matrix, representing the contribution of voxel $i$ to bin $j$, were calculated by including the PSF of the collimator/detector system. As mentioned above, three methods were considered in the modeling of PSF.

The first (referred to as the $\mathrm{G}$ model) involved obtaining $a_{i j}$ by taking into account the geometrical aperture of the pinhole but not considering the intrinsic detector PSF (PSFi) or septal penetration throughout the pinhole collimator. To this end, the center of voxel $i$ was projected onto the surface of the detector and the coordinates of this projection point were determined. A circle centered on these coordinates was then considered, the diameter of which was the projection of the diameter of the pinhole aperture on the detector surface in the direction defined by the center of voxel $i$ and the center of the pinhole. The value of $a_{i j}$ was calculated as follows: (a) If the entire bin was included in the projected circle, the value of $a_{i j}$ was set to 1 . (b) If the bin was only partially included in the projected circle, the value of $a_{i j}$ was set to the fraction of bin included in the projected circle. In all cases, the calculated values of $a_{i j}$ were normalized using a sensitivity value that took into account the penetration through the pinhole collimator ${ }^{19,20}$

$$
S \propto \frac{d^{2}}{16 Z^{2}} \sin ^{x} \theta
$$

where $S$ is the ratio of $\gamma$-rays detected against the total number of $\gamma$-rays emitted isotropically from the source, $\theta$ is the incidence angle measured from the plane of the pinhole, $Z$ is the perpendicular distance from voxel $i$ to the plane of the pinhole, and $d$ is the diameter of the pinhole. A value for the sensitivity exponent $x$ was obtained from experimental acquisitions of sensitivity measurements. The sensitivity exponent $x$ of our equipment was found to be 4.3.

The second approach (referred to as the SP model) included the geometrical aperture of the pinhole collimator and septal penetration at the edges of the pinhole. The septal penetration was modeled following a method proposed by Accorsi and Metzler. ${ }^{17,18}$ The method is geometric and ignores scatter in the collimator. This is not a severe limitation as, according to the literature ${ }^{22}$ for common low energy radioisotopes such as ${ }^{99 m} \mathrm{Tc}$, scattered photons represent only a very small percent of the total number of photons detected. This approach is based on the hypothesis that a resolution-equivalent diameter can be defined as the diameter of an ideal pinhole with the same geometric resolution as the total resolution (geometric plus penetration) of the real pinhole. The radial (parallel to the direction of the tilt of the point source) and transversal (perpendicular to the radial direction) components must be treated differently, as follows: ${ }^{17}$

$$
\begin{aligned}
& d_{\mathrm{re}}^{\mathrm{rad}} \approx d+\Delta L_{k}\left(\tan ^{2}\left(\frac{\alpha}{2}\right)-\cot ^{2} \theta\right) \cot \left(\frac{\alpha}{2}\right) \sin \theta, \\
& d_{\mathrm{re}}^{\mathrm{tra}} \approx \sqrt{\left(d+\Delta L_{k} \tan \left(\frac{\alpha}{2}\right) \sin \theta\right)^{2}-\Delta L_{k}^{2} \cos ^{2} \theta},
\end{aligned}
$$

where $d$ is the physical diameter of the pinhole, $\theta$ the incidence angle measured from the plane of the pinhole, $\alpha$ is the full acceptance angle of a knife-edge pinhole, and $\Delta L_{k}$ is the path length through the collimator that attenuates the number 
of photons by a factor of $k\left(\Delta L_{k} \equiv-\ln k / \mu, \mu\right.$ being the linear attenuation coefficient in the collimator). In this paper, a value of $k=0.5$ was used. ${ }^{5}$ In our pinhole collimator, $\alpha=90^{\circ}$. A linear attenuation coefficient of $\mu=36.3 \mathrm{~cm}^{-1}$ was considered for the $140 \mathrm{keV}$-energy photons of the ${ }^{99 m} \mathrm{Tc}$ in the tungsten alloy of the collimator. Modeling of septal penetration included the radial and transversal effective diameters described in Eqs. (2) and (3) of the G model.

In the third approach (referred to as the SP-PSFi model), detector response (PSFi) was also taken into account in the spatially variant PSF. The spatial resolution $\left(\mathrm{R}_{\mathrm{O}}\right)$ of a pinhole collimator can be approximated by ${ }^{21}$

$$
R_{0}^{2}=\left[R_{\mathrm{PSF}_{i}}\left(\frac{Z}{f}\right)\right]^{2}+\left[d_{e}\left(1+\frac{Z}{f}\right)\right]^{2}
$$

where $R_{\mathrm{PSF}_{i}}$ is the intrinsic resolution of the detector, $f$ is the focal length of the pinhole collimator, $Z$ is the perpendicular distance between the point source and the plane of the collimator, and $d_{e}$ is the effective diameter of the pinhole aperture. This expression clearly illustrates that spatial resolution depends on both the intrinsic resolution of the detector (first term) and the collimator aperture (second term). In high magnification systems, the detector contribution is less than that of the pinhole aperture and its inclusion in the PSF has a low impact on reconstructed images. ${ }^{16}$ However, in low magnification systems, both contributions are similar and the inclusion of intrinsic detector response in PSF modeling can improve the reconstructed image. So, the third method combined the geometrical aperture of the pinhole collimator, detector response, and septal penetration at the edges of the pinhole. To this end, the PSFi was modeled from experimental data and then convolved with the SP model.

The reconstruction algorithm included the geometrical parameters that describe a rotating system, ${ }^{23,24}$ such as focal length $(f)$, electric shifts $\left(e_{u}, e_{v}\right)$ of the image, the ROR, mechanical offset $(m)$, tilt angle $(\Phi)$, and twist angle $(\Psi)$.

Correction for scatter and attenuation in the object was not included in the reconstruction algorithm.

\section{B. Experimental setup}

In this section, we describe the equipment used, the tests performed to calculate calibration parameters, and the measurement of PSFi for different incidence angles.

\section{B.1. SPECT system}

Figure 1 shows the SPECT system ${ }^{23}$ based on a Sentinella S102 gamma camera (Oncovision, Valencia, Spain) ${ }^{25}$ equipped with a $\mathrm{CsI}(\mathrm{Na})$ continuous crystal detector of $4.0 \mathrm{~mm}$ thickness covering an effective detection area of 40 $\times 40 \mathrm{~mm}^{2}$. This detector area was binned by default into $300 \times 300$ pixels with a pixel size of $0.13 \times 0.13 \mathrm{~mm}^{2}$. The gamma camera had an energy resolution of $13 \%$ at $140 \mathrm{keV}$. A pinhole collimator with a focal length of $32 \mathrm{~mm}$ and a hole of $1 \mathrm{~mm}$ in diameter was employed.

The gantry consisted of a $20 \mathrm{~cm}$ radius metallic disc controlled with a motorized rotary stage (OWIS GmbH,

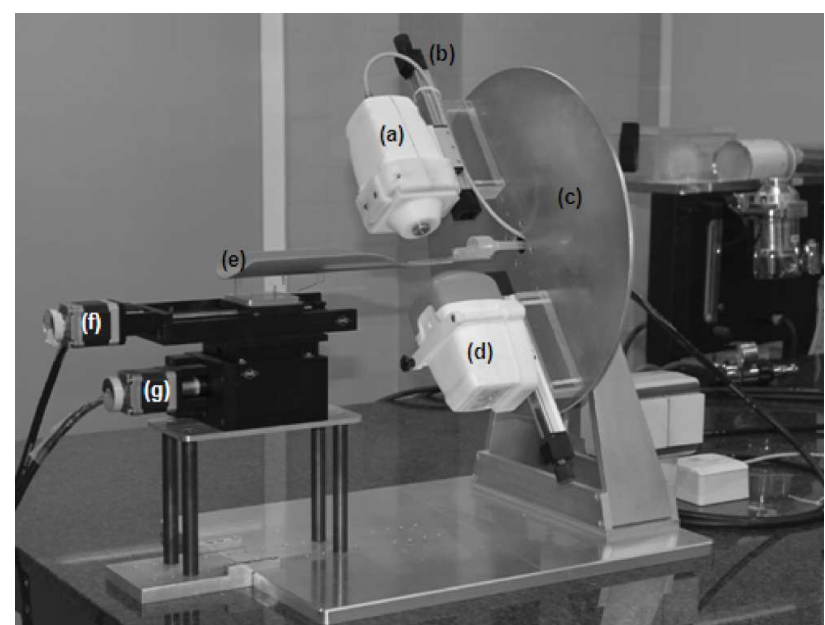

FIG. 1. Pinhole SPECT imaging system consisting of a gamma camera (a), a micrometric positioning system (b), a rotor (c), a counterbalance (d), a bed (e), a positioning system (f), and a motorized elevator ( $g$ ).

Staufen, Germany). A manually controlled micrometric linear positioning system (range: $20-140 \mathrm{~mm}$ ) allowed us to select the most suitable ROR. The second gamma camera currently has a counterweight function to balance the gantry. Two motorized linear stages (OWIS GmbH, Staufen, Germany) controlled horizontal and vertical displacements of a bed $15 \mathrm{~cm}$ long for animal positioning. Multiple bed SPECT acquisitions were implemented through automatic bed movement.

\section{B.2. Intrinsic PSF detector measurement}

The dependence of PSFi parameters on the incidence angle on the detector surface was measured using a collimated pencil beam generated from a point source of ${ }^{99 m} \mathrm{Tc}$ at one end of a $30 \mathrm{~cm}$ long shielded tube with an aperture of $0.5 \mathrm{~mm}$ at the other end. The measures of the intrinsic PSF were carried out very close to the detector surface to avoid beam divergence, and at the same distance from the surface for all the angles of incidence.

Experimental measurements were carried out at $10^{\circ}$ intervals from $90^{\circ}$ (incidence perpendicular to the detector) to $40^{\circ}$. The PSFi can be modeled using an asymmetric ${ }^{26}$ rather than symmetric ${ }^{15} 2 \mathrm{D}$ Gaussian distribution. For a pencil beam with an angle of incidence other than $90^{\circ}$, Gaussian distribution is defined by three parameters: the asymmetric inner and outer radial components (parallel to the direction of tilt of the point source) and the symmetric transversal component (perpendicular to the radial direction). Four images were taken for each incidence angle, locating the beam in different parts of the camera's field of view (FOV). Asymmetrical 2D Gaussian distributions were fitted to each PSFi, thus obtaining the standard deviations $\sigma_{T}, \sigma_{\mathrm{Ri}}, \sigma_{\mathrm{Ro}}$, respectively. In order to model the PSFi, a second order polynomial was fitted to each component as a function of the incidence angle.

\section{C. Resolution}

Resolution measurements were made using two different phantoms to assess the tomographic resolution for different 
ROR and to evaluate the capability of separating rods at different distances apart. Image reconstruction was performed using the three PSF models described above (G, SP, and SP-PSFi). A reconstruction scheme of five subsets for 60 projections every $6^{\circ}$, with a bin size of $0.78 \times 0.78 \mathrm{~mm}^{2}$, was used. Depending on ROR, different dimensions were considered for voxel size of the reconstructed images.

\section{C.1. Hot-rod phantom}

A hot-rod-in-air phantom was used to measure the tomographic resolution of the system. This phantom contained four capillaries of $60 \mathrm{~mm}$ length and $0.3 \mathrm{~mm}$ inner diameter, placed in air. The capillaries were set parallel to the axis of rotation of the system at $1.3,4.8,8.3$, and $11.4 \mathrm{~mm}$ from the center of the FOV and followed a spiral distribution, allowing the tomographic resolution to be assessed as a function of the distance from the center. These distances were chosen to cover the FOV determined by the ROR used in a small-animal study. Capillaries were filled with $37 \mathrm{MBq} / \mathrm{mL}(1 \mathrm{mCi} / \mathrm{mL})$ of a ${ }^{99 m} \mathrm{Tc}$ solution. In order to evaluate the tomographic resolution as a function of the distance to the center, 60 projections of $30 \mathrm{~s}$ each over $360^{\circ}$ were acquired for ROR of $21,27,32$, and $37 \mathrm{~mm}$. A $120 \times 120 \times 40$ matrix size and a $0.25 \times 0.25 \times 1.00 \mathrm{~mm}^{3}$ voxel size were used in reconstruction.

For each slice, the radial direction was considered as that defined by the center of the FOV and the center of the hot rod and the transversal was taken to be perpendicular to the radial direction. Transversal and radial FWHMs were obtained by fitting 2D Gaussian distributions to each slice of the reconstructed hot rod, to take into account the behavior in the transversal and radial axes and averaging the values obtained over a $5 \mathrm{~mm}$ length.

\section{C.2. Hot-rod sector phantom}

In order to assess any improvements in reconstructed images, an in-house phantom was used. The same scheme as that described above (five subsets for 60 projections every $6^{\circ}$, and a bin size of $0.78 \times 0.78 \mathrm{~mm}^{2}$ ) was employed in reconstruction. The phantom contained 22 capillaries $60 \mathrm{~mm}$ in length with an inner diameter of $0.3 \mathrm{~mm}$, placed in air and arranged in $120^{\circ}$ sectors. Distances between capillaries were $3 \mathrm{~mm}$ (four capillaries), $2 \mathrm{~mm}$ (nine capillaries), and $1.5 \mathrm{~mm}$ (nine capillaries) depending on the sector. Capillaries were filled with $37 \mathrm{MBq} / \mathrm{mL}(1 \mathrm{mCi} / \mathrm{mL})$ of a ${ }^{99 m} \mathrm{Tc}$ solution. A ROR of $14.0 \mathrm{~mm}$ and an acquisition time of $60 \mathrm{~s} /$ projection were employed. In the reconstruction, $80 \times 80 \times 20$ matrix size and $0.20 \times 0.20 \times 0.80 \mathrm{~mm}^{3}$ voxel size were used.

\section{D. Noise, recovery coefficients, and contrast}

Contrast, recovery coefficients, and noise were quantified using a specially designed NEMA NU 4-2008 (Ref. 27) image-quality phantom. The phantom is made of polymethylmethacrylate with internal dimensions of $50 \mathrm{~mm}$ length and $30 \mathrm{~mm}$ diameter. It consists of three parts. (1) The main phantom body comprises a fillable cylindrical chamber of
$30 \mathrm{~mm}$ diameter and $30 \mathrm{~mm}$ length. The remaining $20 \mathrm{~mm}$ length of phantom body is solid with five fillable rods drilled through (at $7 \mathrm{~mm}$ from the center) with diameters of 1, 2, 3 , 4, and $5 \mathrm{~mm}$, respectively. (2) A lid attaches to the large uniform region end of the phantom and supports two region chambers. These chambers are hollow cylinders $15 \mathrm{~mm}$ in length and $8 \mathrm{~mm}$ in diameter. (3) A lid on the opposite side of the phantom facilitates drying and the removal of trapped air.

One chamber was filled with $37 \mathrm{MBq} / \mathrm{mL}(1 \mathrm{mCi} / \mathrm{mL})$ of a ${ }^{99 m} \mathrm{Tc}$ solution (hot chamber), which was twice the concentration of the main body region $(18.5 \mathrm{MBq} / \mathrm{mL})$. The other independent chamber was air-filled (cold chamber). Five phantom acquisitions were performed, with a ROR of $33.8 \mathrm{~mm}$ and an acquisition time of $30 \mathrm{~s} /$ projection. In order to compare the methods under optimal conditions, the sum of the five acquisitions was used in reconstruction. A matrix size of $110 \times 110 \times 60$ and voxel size of $0.35 \times 0.35 \times 0.70 \mathrm{~mm}^{3}$ were used.

\section{D.1. Noise}

The uniform region of the NEMA NU 4-2008 phantom was used to characterize image noise, ${ }^{27}$ expressed as the percentage standard deviation $\left(\% \mathrm{STD}_{\text {unif }}\right)$ in a central cylindrical volume of interest, $22.5 \mathrm{~mm}$ in diameter $(75 \%$ of active diameter) and two slices in length.

\section{D.2. Recovery coefficients}

$\mathrm{RC}$ were calculated for each fillable rod $i$ as

$$
\mathrm{RC}_{i}=\left(\frac{C_{i}}{C_{B}}\right) \cdot 100 \%,
$$

where $\mathrm{C}_{i}$ is the average counts in a ROI of $5 \mathrm{~mm}$ length and 1 , $2,3,4$, and $5 \mathrm{~mm}$ diameter within the $\operatorname{rod} i$ region; $\mathrm{C}_{\mathrm{B}}$ is the average counts in the ROI of $22.5 \mathrm{~mm}$ diameter $(75 \%$ total active diameter) and two slices in length, within the uniform region.

CNR was calculated for each fillable rod $i$ as

$$
\mathrm{CNR}_{i}=\left(\frac{\mathrm{RC}_{i}}{\% \mathrm{STD}_{\text {unif }}}\right) \text {. }
$$

\section{D.3. Contrast}

Contrast was quantified using measurements in the uniform region and in the two independent chambers of the phantom. The percent contrast $Q_{H}$ for the hot chamber was calculated according to the definition

$$
Q_{H}=\frac{C_{H} / C_{B}}{a_{H} / a_{B}} \cdot 100 \%,
$$

where $C_{H}$ is the average counts in a ROI of $5 \mathrm{~mm}$ length and $8 \mathrm{~mm}$ diameter within the hot chamber, $C_{B}$ is the average counts in the ROI of $22.5 \mathrm{~mm}$ diameter $(75 \%$ total active diameter) and two slices in length within the uniform region, $a_{H}$ is the activity concentration in the hot chamber, and $a_{B}$ is the activity concentration in the uniform region. The percent contrast $Q_{C}$ for the cold chamber was calculated for each 

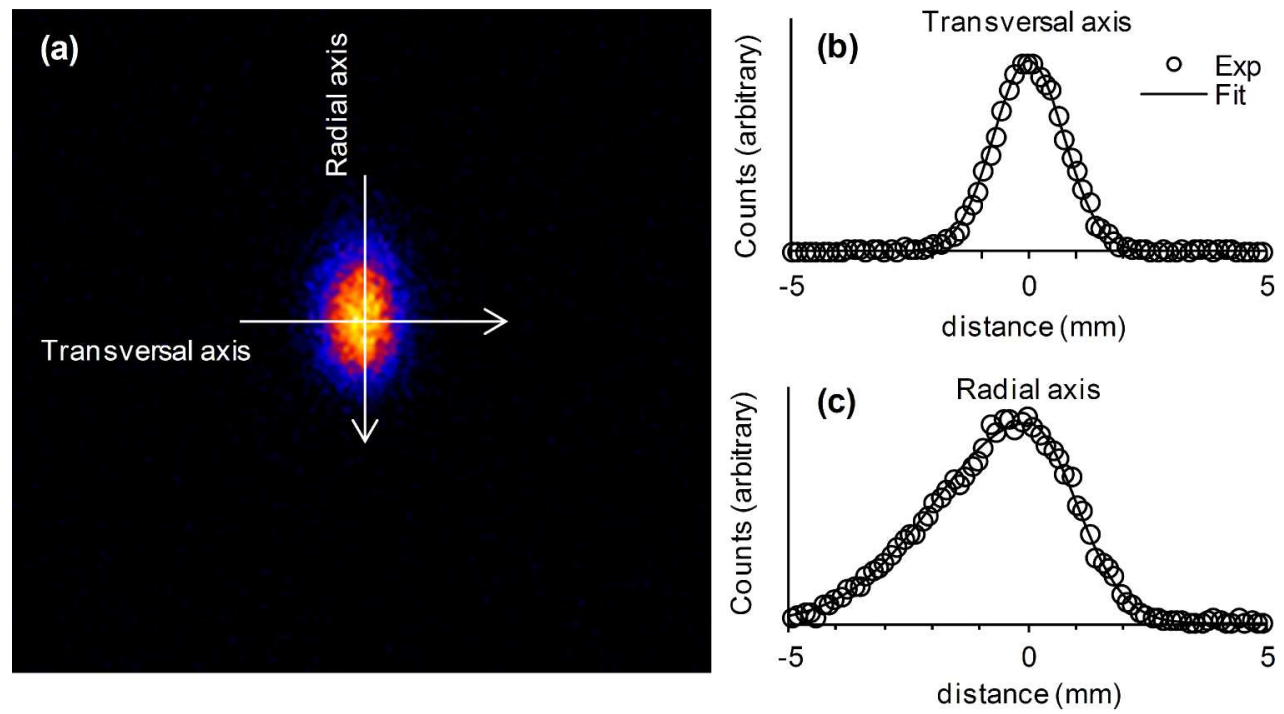

FIG. 2. (a) Intrinsic detector PSF image at a $40^{\circ}$ angle of incidence, (b) radial, and (c) transversal collapsed experimental profiles and fitted Gaussian distributions.

acquisition as

$$
Q_{C}=\left(1-\frac{C_{C}}{C_{B}}\right) \cdot 100 \%,
$$

where $C_{C}$ is the average counts in a ROI of $5 \mathrm{~mm}$ length and $8 \mathrm{~mm}$ diameter within the cold chamber and $C_{B}$ is the average counts in the ROI of $22.5 \mathrm{~mm}$ diameter $(75 \%$ total active diameter) and two slices in length within the uniform region.

\section{E. Animal study}

A head bone scan of a $30 \mathrm{~g}$ weight CD1 mouse was performed using a two-bed acquisition scheme (10 $\mathrm{mm}$ apart) with a ROR of $19.5 \mathrm{~mm}$ and an acquisition time of 60 s/projection. To this end, $355 \mathrm{MBq}(9.6 \mathrm{mCi})$ of ${ }^{99 m} \mathrm{Tc}$ hydroxymethane diphosphonate $\left({ }^{99 m} \mathrm{Tc}-\mathrm{HDP}\right)$ was injected into the mouse tail vein. The SP-PSFi model and a matrix size of $100 \times 100 \times 50$ and voxel size of $0.25 \times 0.25 \times 1.00 \mathrm{~mm}^{3}$ were used in the reconstruction.

The experimental work complied with the Spanish legislation on the "Protection of Animals used for Experimental and other Scientific Purposes," and with the Directives of the European Union. The animal was anesthetized before tracer injection.

\section{RESULTS}

\section{A. Intrinsic detector PSF measurements}

Figure 2(a) shows the PSFi obtained when the angle of incidence of the collimated pencil beam was $40^{\circ}$. The collapsed profiles in the transversal and radial axes are shown in Figs. 2(b) and 2(c), respectively.

An excellent agreement was found between the experimental data and the fitted values. Figure 3 summarizes the fitted values obtained for $\sigma_{T}, \sigma_{\mathrm{Ri}}$, and $\sigma_{\mathrm{Ro}}$ when varying the incidence angle of the collimated pencil beam. While the transversal component is almost invariant with incidence angle, it can be seen how standard deviations in both inner and outer radial components increase as the incidence angle decreases, with the inner standard deviation of the radial axis being smaller than the outer one. This increment was expected because of the path of photons through the continuous crystal for an incidence angle of less than $90^{\circ}$. As plots in Fig. 3 show, a second-order polynomial fit is suitable to characterize the dependence of the inner and outer radial components on the incidence angle. The transversal component was assumed to be independent of the incidence angle.

\section{B. Resolution}

\section{B.1. Tomographic resolution as a function of $R O R$}

Figure 4 shows the relationship between tomographic resolution at the center of the FOV and iterations for different ROR (by varying the PSF model). In this case, because of the

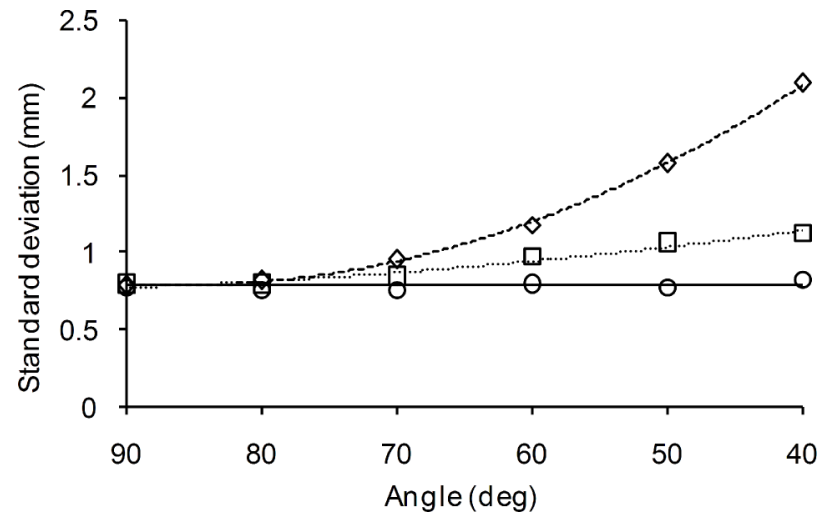

FIG. 3. Inner $(\square)$ and outer $(\diamond)$ radial and transversal $(\bigcirc)$ standard deviations of the intrinsic detector PSF obtained from experimental data for each angle considered. Second-order polynomial fitting for inner and outer components and horizontal line for transversal component are displayed in solid line. 
(a)

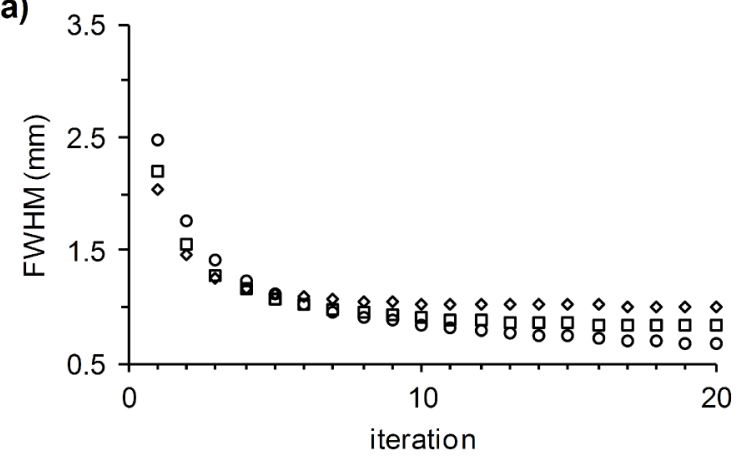

(c)

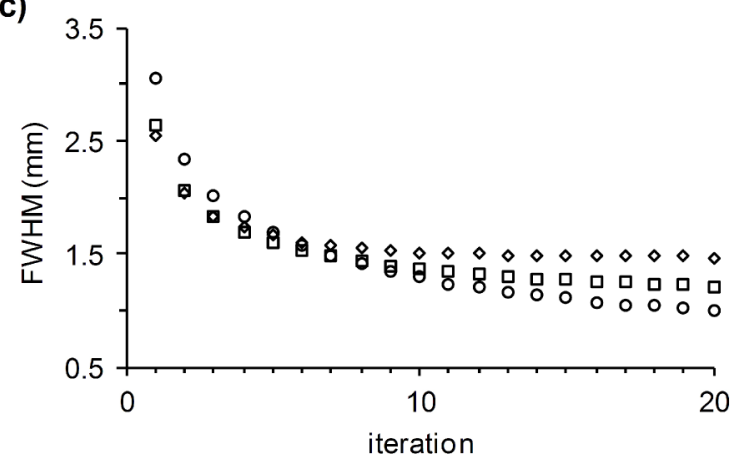

(b)

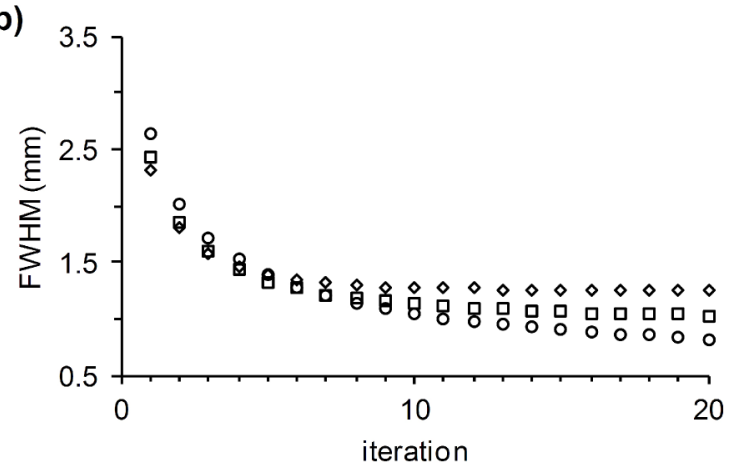

(d)

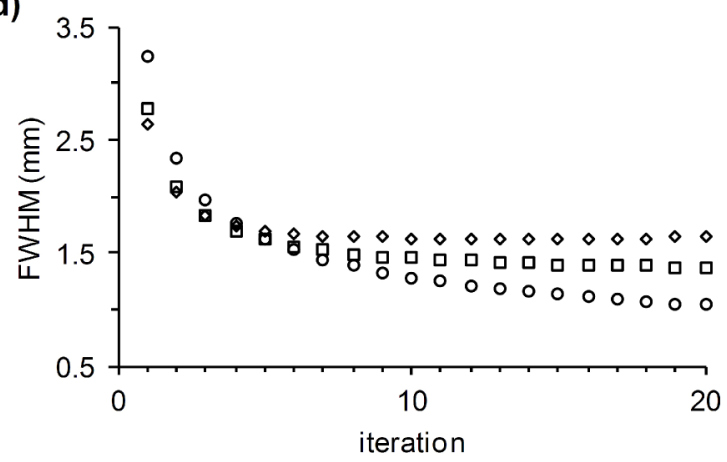

FIG. 4. FWHM at the center of the FOV as a function of the number of iterations for an acquisition radius of 21 (a), 27 (b), 32 (c), and $37 \mathrm{~mm}$ (d) and for G ( $\diamond)$, SP (ם), and SP-PSFi (O) models.

symmetry of the image of the hot rod located at the center of the FOV, radial and transversal resolution components were not calculated separately. Thus, a single value of resolution was calculated by fitting a symmetric Gaussian distribution to the hot rod reconstructed image. A decrease of FWHM with iterations was observed for all three PSF models. For a low number of iterations (up to five), FWHM value was the lowest in the G model, followed by SP and SP-PSFi for the same number of iterations. This behavior was observed for all ROR. After iteration 6, the order reverses, with FWHM lowest in (a)

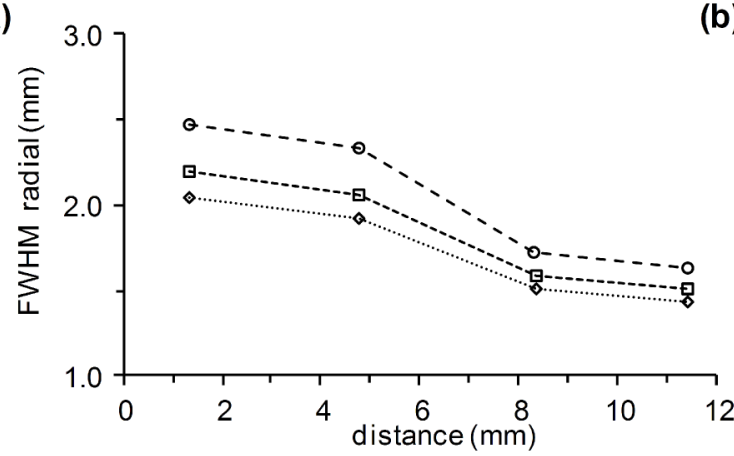

(c)

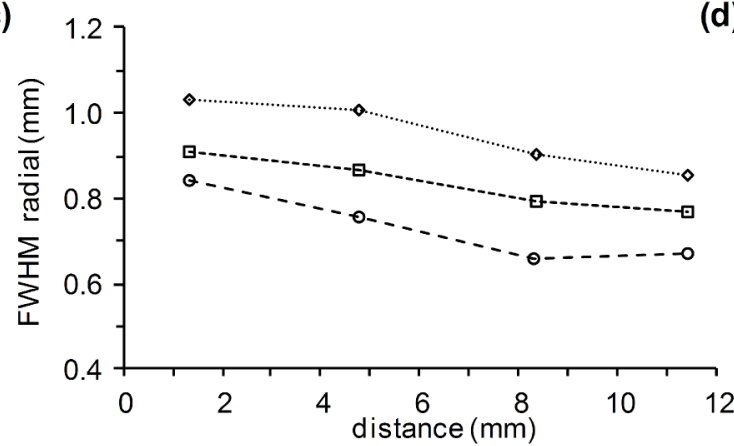

(b)

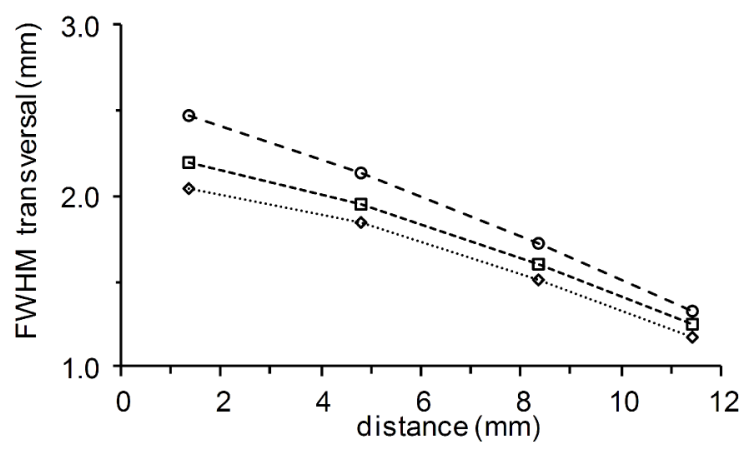

(d)

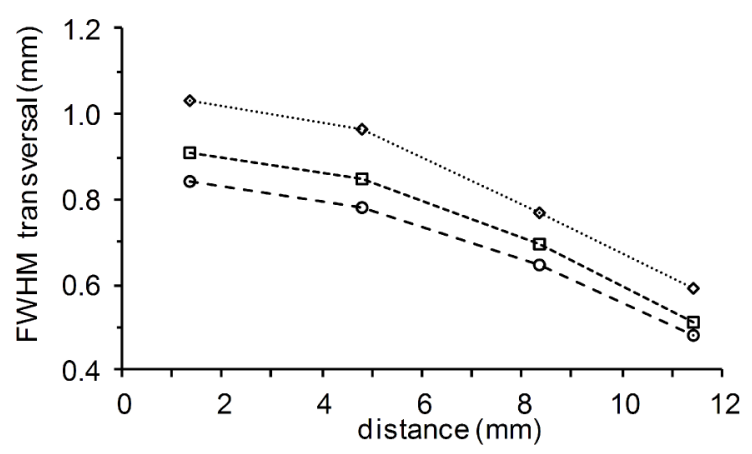

Fig. 5. Radial and transversal FWHM dependence on distance from the origin of the image for an acquisition radius of $21 \mathrm{~mm}$ for G ( $\diamond)$, SP ( $\square$ ), and SP-PSFi (O) models. (a) and (c) Radial resolution for 1, 10 iterations. (b) and (d) Transversal resolution for 1, 10 iterations. 
G

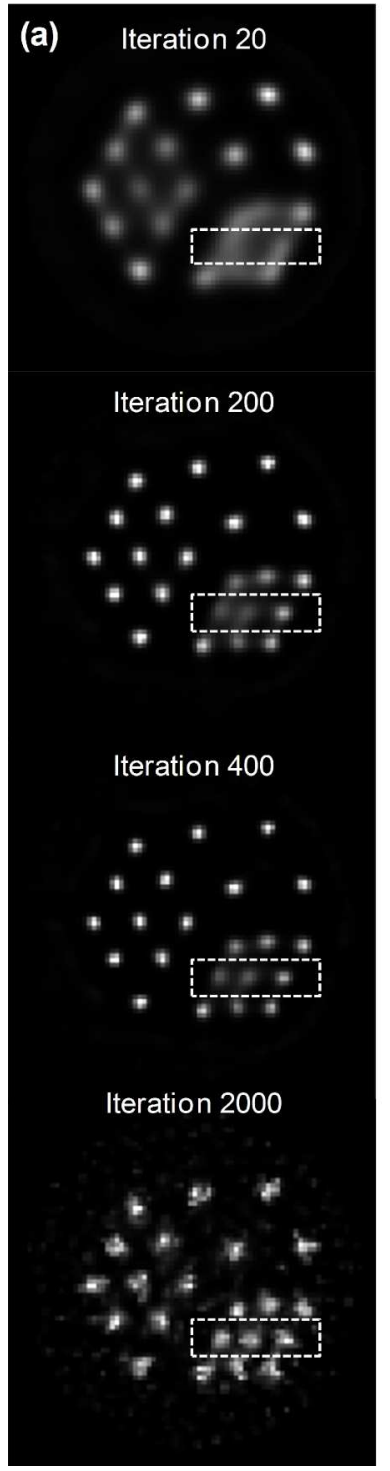

SP

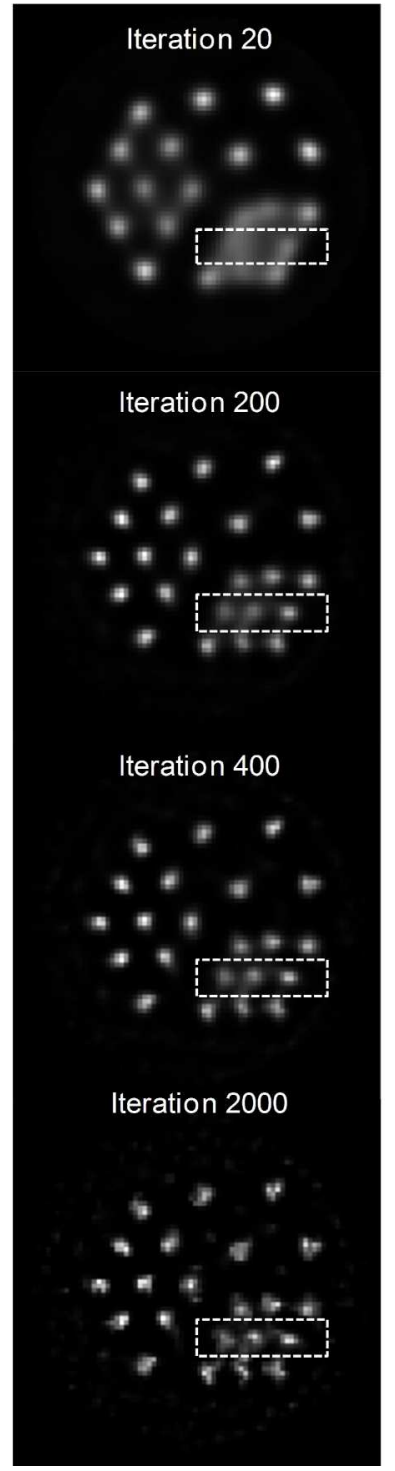

SP-PSFi

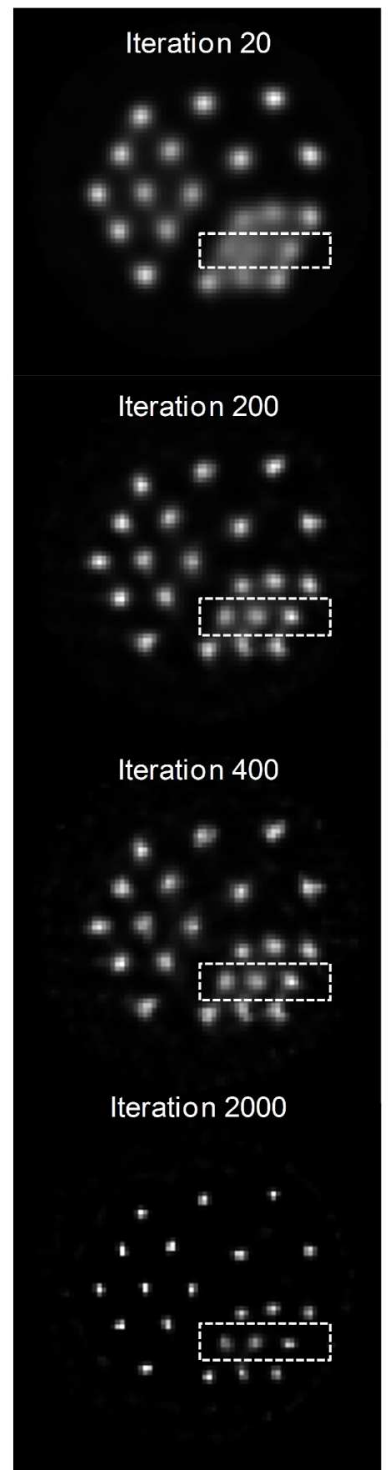

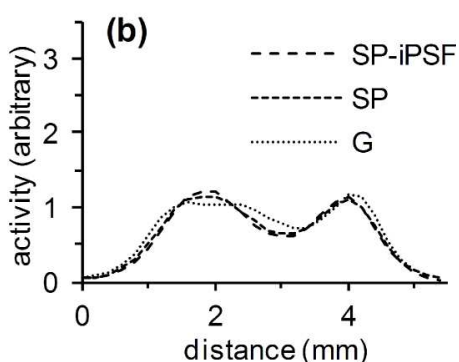
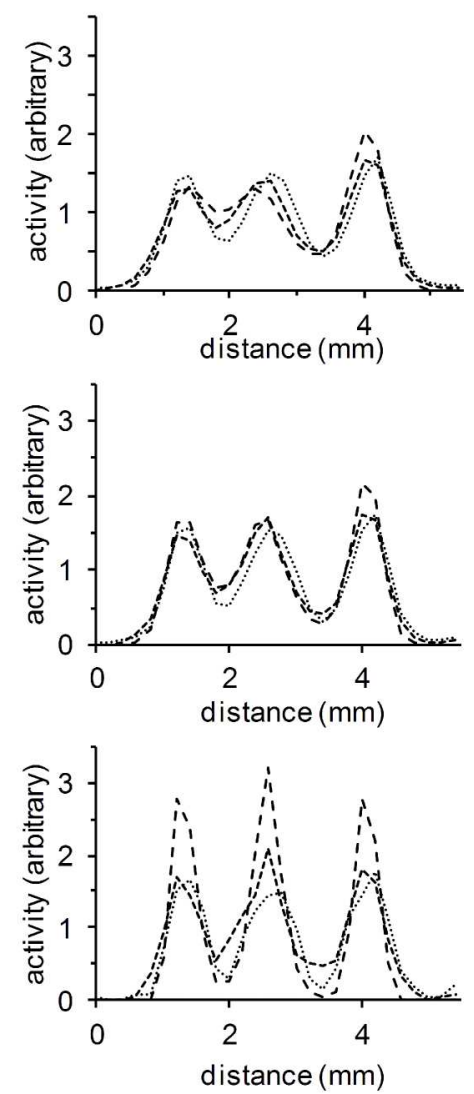

FIG. 6. (a) Sector phantom reconstruction for SP-PSFi (left), SP (middle), and G (right) models for iterations 20, 200, 400, and 2000. (b) Profile of 1.5 mm sector.

the SP-PSFi model, followed by SP and G. FWHM reaches a plateau for $\mathrm{G}$ model around iteration 6 for all ROR. The value of the plateau depends on the ROR, being greater for higher RORs. This is due to the fact that the term of intrinsic PSF in Eq. (4) (that was not included in $G$ model) becomes more important as ROR increases. In the case of SP model, the behavior of FWHM with iterations is similar to that of $\mathrm{G}$ model, but the plateau value is lower. Finally, for SP-PSFi model, convergence is not achieved at iteration 20. In this case, the FWHM value obtained at this iteration is lower for lower ROR.

\section{B.2. Tomographic resolution as a function of the distance from the center of the FOV}

Figure 5 shows the relationship between the radial and transversal tomographic resolution (measured as the FWHM of the reconstructed image of a hot rod) and the distance from the hot rod to the center of the FOV (for the different PSF models). A decrease in FWHM values is observed for both components when the distance from the center of the FOV increases.

At iteration 1, the lowest value of FWHM was obtained for $\mathrm{G}$ model, followed by SP and SP-PSFi. At iteration 10, the order reverses, with FWHM lowest in the SP-PSFi model, followed by SP and G.

\section{B.3. Hot-rod sector phantom}

Figure 6(a) shows an axial slice of the reconstructed images of the sector phantom for G, SP, and SP-PSFi models and for iterations 20,200, 400, and 2000. Profiles in the central row of the $1.5 \mathrm{~mm}$ sector are shown in Fig. 6(b) for G, SP, and SP-PSFi models and for all iterations. Profiles showed a faster convergence for $\mathrm{G}$ and SP models, but with better convergence in high iterations in the case of SP-PSFi model. 


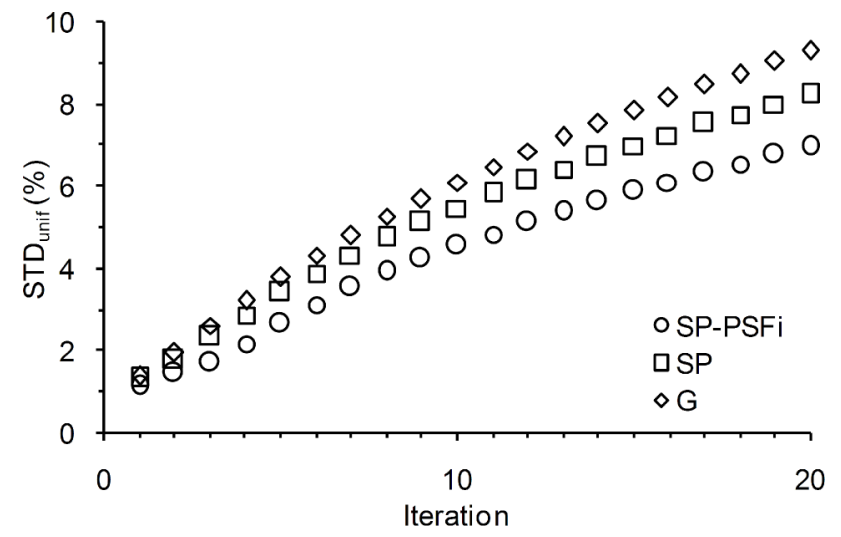

FIG. 7. $\% \mathrm{STD}_{\text {unif }}$ dependence with iterations for $\mathrm{G}(\diamond)$, SP ( () , and SP-PSFi ( 0 ) models.

\section{C. Noise, recovery coefficients, and contrast}

\section{C.1. Noise in uniform phantom region}

Figure 7 shows the relationship between the noise in uniform phantom region $\% \mathrm{STD}_{\text {unif }}$ and iterations for the three PSF models. As expected, the noise increased with the number of iterations for all models, with the greatest increase seen in the G model, followed by SP and SP-PSFi.

\section{C.2. Recovery coefficients}

Figure 8 shows an axial slice of the NEMA NU 4-2008 image-quality phantom containing five hot rods of different diameters for G, SP, and SP-PSFi models. The number of iterations for G, SP, and SP-PSFi models was chosen so that a comparison between the corresponding recovery coefficients could be made using images with similar signal-to-noise ratio. For a low number of iterations (two first columns, i.e., until five iterations for SP-PSFi model), images are smooth for all three PSF models, and no relevant visual differences are observed. A number of iterations higher than ten for SP-PSFi model (and equivalent \%STD iterations for SP and G models) resulted in the appearance of a ring-type artifact in the $5 \mathrm{~mm}$ rod for all PSF models. For these iterations, all rods of a diameter greater than $1 \mathrm{~mm}$ are clearly visible, but this is not the case for the $1 \mathrm{~mm}$ diameter rod.

Figure 9 (a) shows profiles of $5 \mathrm{~mm}$ rod reconstructed image with the SP-PSFi model of Fig. 8(a). As can be observed, the maximum pixel value is not recovered at iteration 1 , while for iteration 5, this value exceeds the true value. For a high number of iterations (over 15), the ring-type artifact is clearly visible. Figure 9(b) shows a zoom of profiles over the region of interest for a number of iterations in the range. ${ }^{9,13}$ Iterations 10 or 11 approximate the values of the reconstructed (a)
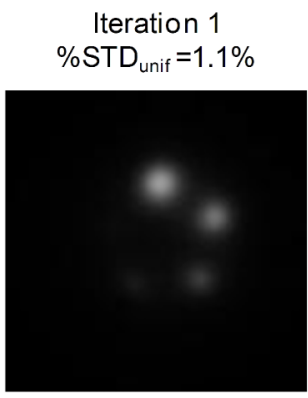

(b)

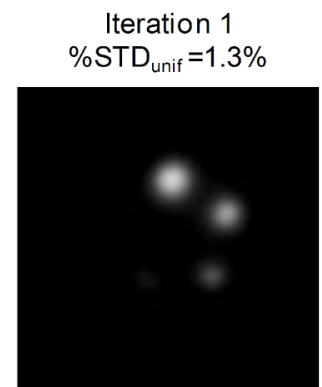

(c)

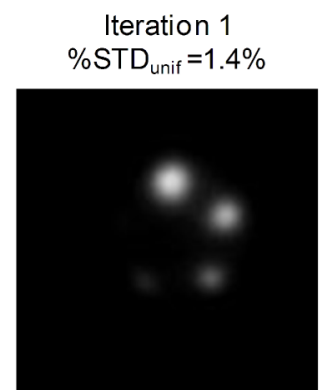

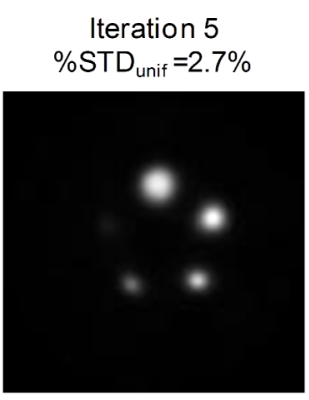

Iteration 4

$\%$ STD $_{\text {unif }}=2.9 \%$

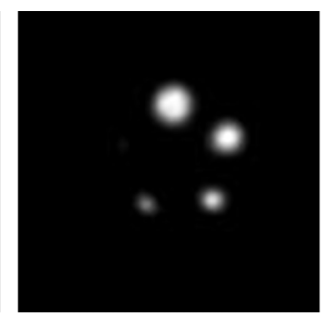

Iteration 3 $\%$ STD $_{\text {unif }}=2.6 \%$

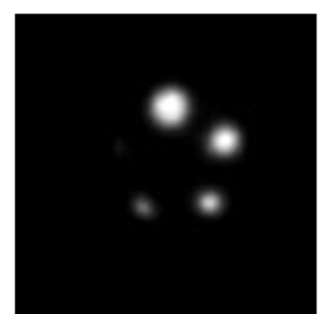

Iteration 10 $\%$ STD $_{\text {unif }}=4.6 \%$

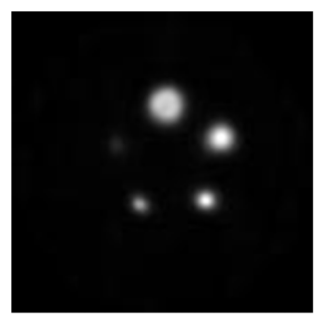

Iteration 8

$\%$ STD $_{\text {unif }}=4.7 \%$

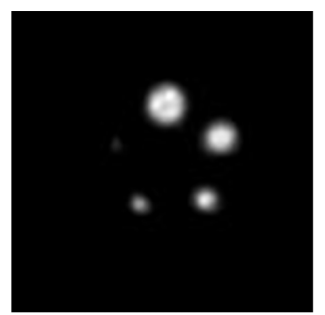

Iteration 7 $\%$ STD $_{\text {unif }}=4.8 \%$

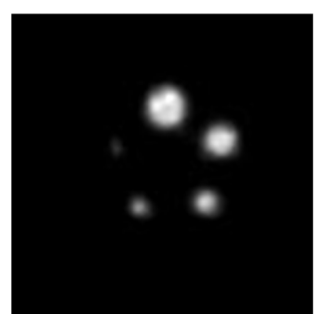

Iteration 15 $\%$ STD $_{\text {unif }}=5.9 \%$

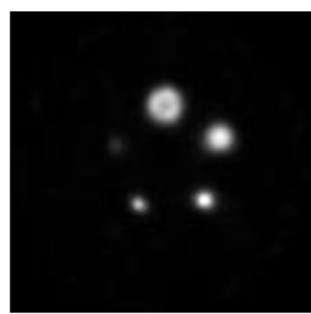

Iteration 11 $\%$ STD $_{\text {unif }}=5.8 \%$

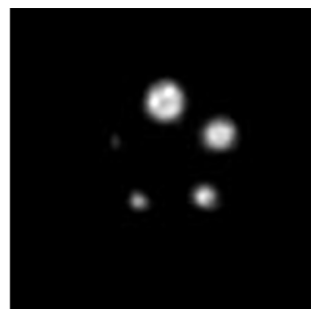

Iteration 9 $\% \mathrm{STD}_{\text {unif }}=5.7 \%$

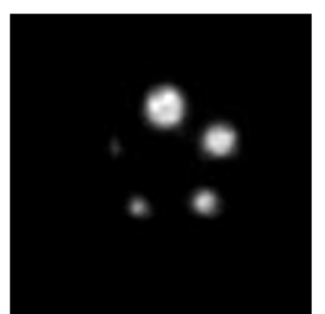

Iteration 20 $\%$ STD $_{\text {unif }}=7.0 \%$

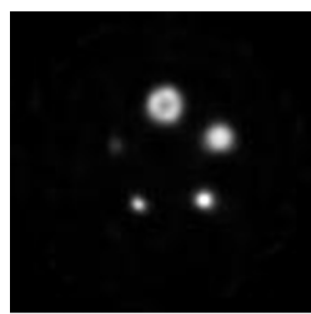

Iteration 15 $\%$ STD $_{\text {unif }}=7.0 \%$

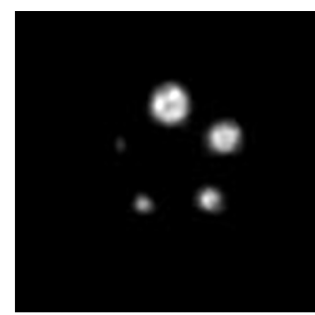

Iteration 12 $\% \mathrm{STD}_{\text {unif }}=6.9 \%$

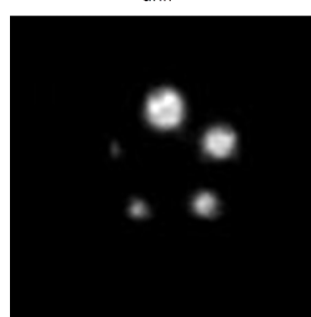

FIG. 8. Axial slice of the NEMA NU 4-2008 reconstructed image, showing rods of 5, 4, 3, 2, and 1 mm diameter. The number of iterations in each column was chosen to compare images with similar noise values for SP-PSFi (a), SP (b), and G model (c). 


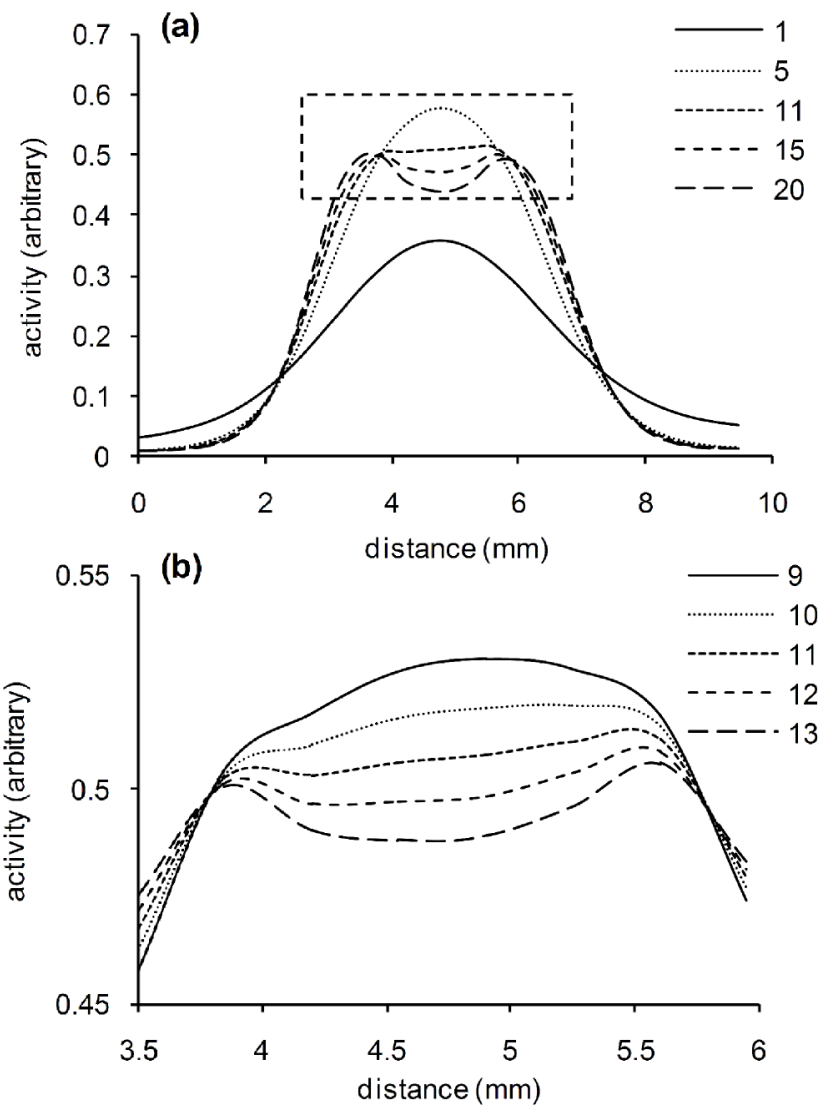

FIG. 9. (a) Profiles of $5 \mathrm{~mm}$ rod reconstructed image with SP-PSFi model for $1,5,11,15$, and 20 iterations. (b) Zoom of reconstructed profiles for 9, 10, 11,12 , and 13 iterations.

image to the theoretical values without the ring-type artifact appearing.

Figure 10 shows axial slices $2.8 \mathrm{~mm}$ apart of the NEMA NU 4-2008 reconstructed image after ten iterations using the SP-PSFi model. The number of iterations was chosen to avoid ring-type artifacts, and the distance between slices was chosen to cover the central region of the FOV $(11.2 \mathrm{~mm})$ in the axial direction.

Figures 11(a)-11(e) show a quantitative comparison of the previous images in terms of $\mathrm{RC}_{\text {rod }}$ as a function of $\% \mathrm{STD}_{\text {unif }}$. As expected, $\mathrm{RC}_{\text {rod }}$ increases with the number of iterations and the rod diameter for all PSF models. For $G$ and SP models, $\mathrm{RC}_{\text {rod }}$ values achieve a convergence value for all rod diameters except for $1 \mathrm{~mm}$. This convergence value depends on the rod diameter and is slightly better for SP model. In the case of SP-PSFi model, convergence is not achieved after 20 iterations for any rod diameter. For this model, convergence is farther when rod diameter is smaller. $\mathrm{RC}_{\mathrm{rod}}$ values obtained for all rod diameters are better for SP-PSFi model than for
$\mathrm{G}$ and SP models for the same level of noise. Figure 11(f) shows the value of CNR as a function of the rod diameter for a $\% \mathrm{STD}_{\text {unif }}$ of approximately $4.7 \%$ (see images in the third column in Fig. 8). This value of $\% \mathrm{STD}_{\text {unif }}$ was attained at iterations 10,8 , and 7 for SP-PSFi, SP, and G models. The number of iterations was chosen to avoid ring-type artifacts. This figure shows higher values of CNR for SP-PSFi for all rod diameters except for that of $1 \mathrm{~mm}$ for which $\mathrm{CNR}$ is similar for all PSF models.

\section{C.3. Contrast quantification}

Figure 12 shows $Q_{H}$ and $Q_{C}$ as a function of $\% \mathrm{STD}_{\text {unif }}$ for all PSF models. The percent contrast values obtained for $\mathrm{G}$ and SP models were considerably lower than in SP-PSFi model for both hot and cold chambers for the same level of noise.

\section{D. Animal study}

Figure 13 shows an axial (a), sagittal (b), and coronal (c) slices of a ${ }^{99 m} \mathrm{Tc}-\mathrm{HDP}$ mouse head scan after ten iterations using the SP-PSFi model. The images show the tracer uptake in the different bone structures.

\section{DISCUSSION}

This work is focused on measuring the improvement of image-quality parameters in pinhole SPECT when corrections are successively incorporated into PSF models. As our results show, the PSFi has a great impact on all the image-quality parameters studied. This impact is clearly higher than that observed in high magnification systems. ${ }^{15,16}$

The first image-quality parameter studied was resolution, and we assessed how resolution depends on the position of the point source in the FOV and on the ROR. To this end, and to facilitate the experimental setup, we used rod sources in air, taking advantage of the published results of Kappadath. ${ }^{31}$ These authors observed small differences between measuring sources in air and in cold background when 3D OSEM algorithm was used, thereby indicating that our measurements using hot rods in air are a good approximation of resolution behavior.

As Fig. 4 shows, the convergence of the algorithm with iterations is slower when more accurate corrections are included in PSF. For iterations higher than five, FWHM values are better when PSF model is more accurate, the behavior being independent on ROR. Our findings demonstrate that in order to improve resolution: (a) an accurate PSF model is
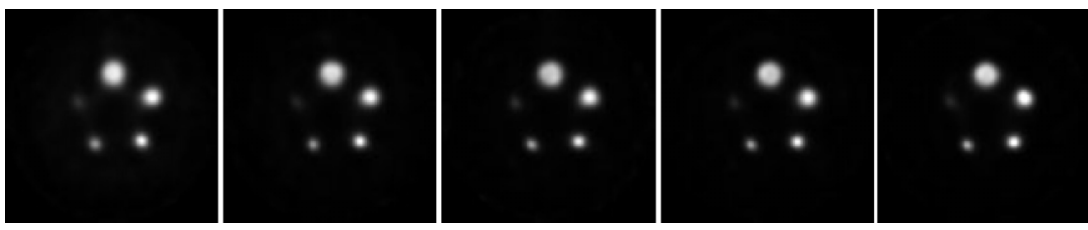

Fig. 10. Axial slices of the NEMA NU 4-2008 reconstructed image with the SP-PSFi after ten iterations. The distance between slices is 2.8 mm. 

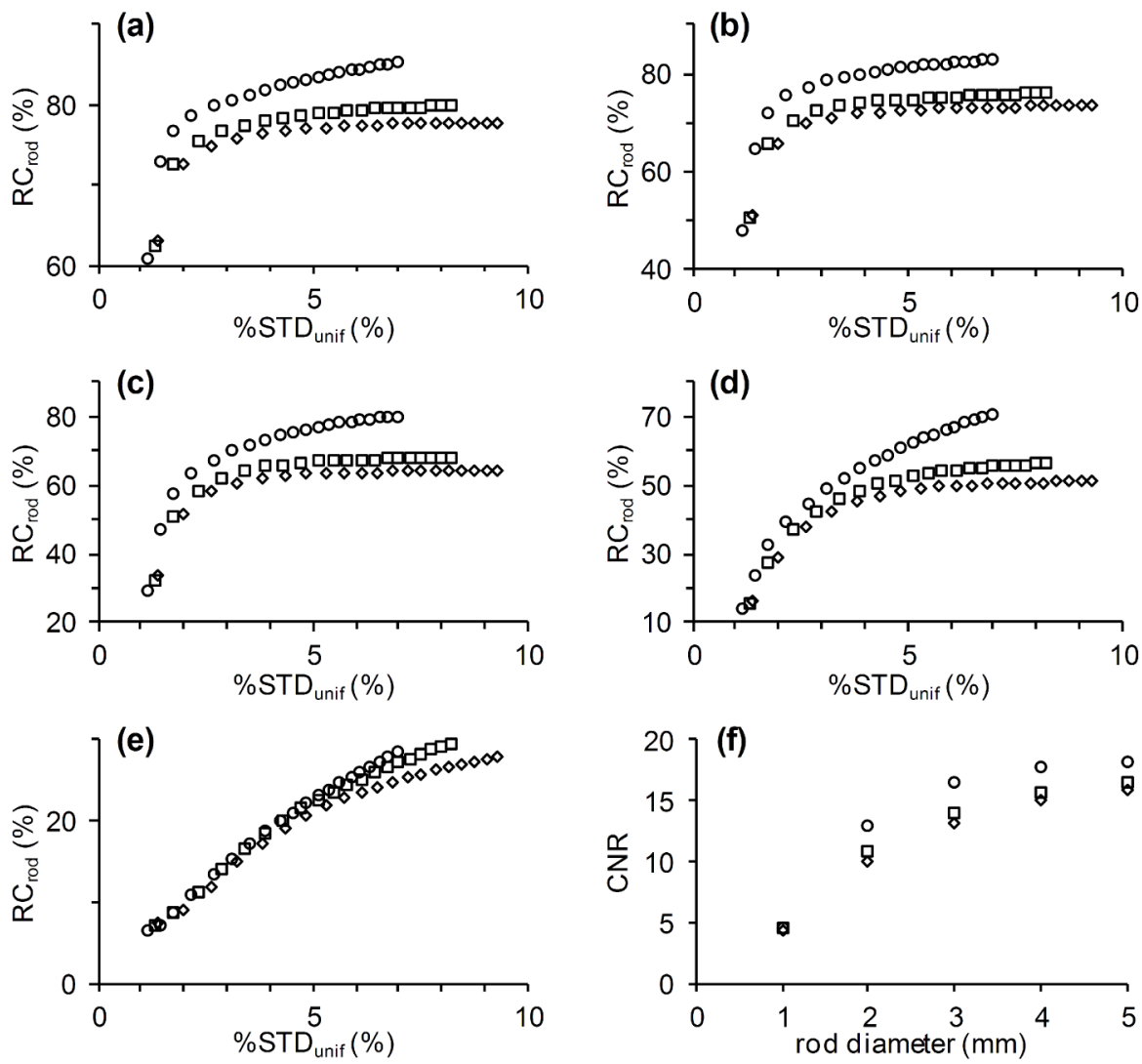

FIG. 11. Recovery coefficient as a function of $\% \mathrm{STD}_{\text {unif }}$ for $\mathrm{G}(\diamond), \mathrm{SP}(\square)$, and SP-PSFi (O) models and for rod diameters of 5 (a), 4 (b), 3 (c), 2 (d), and $1 \mathrm{~mm}$ (e). (f) Contrast-to-noise ratio as a function of rod diameter for a noise level of $4.7 \%$ in the uniform region of the NEMA NU 4-2008 phantom, corresponding to iterations 10, 8, and 7 for SP-PSFi, SP, and G models, respectively.
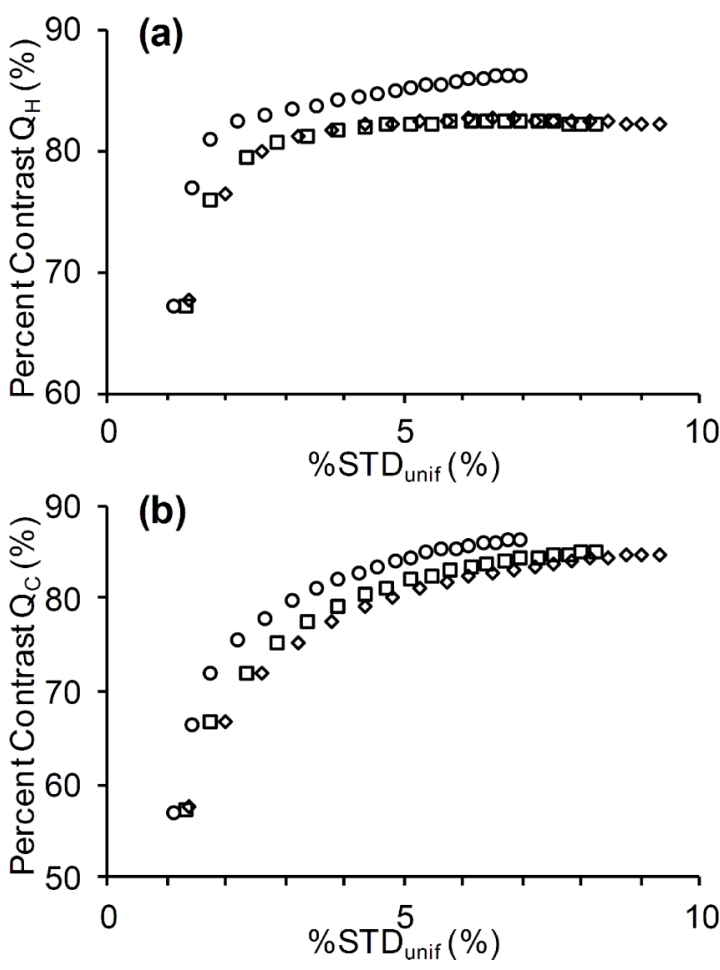

FIG. 12. Percent contrast as a function of $\% \mathrm{STD}_{\text {unif }}$ for G $(\diamond)$, G-PSFi ( $\square$ ), and SP-PSFi (O) models for (a) hot cylinder and (b) cold cylinder. necessary, (b) the inclusion of PSFi plays a major role, and (c) a minimum number of five iterations are required.

In Fig. 5, we can see that the FWHM decreases in both transversal and radial components from the center to the periphery for all PSF models. We found that for images of the same digitized phantom, projected and reconstructed using the SP-PSFi model, for a very high number of iterations FWHM values did not depend on position in the FOV or the acquisition ROR. These findings would indicate that the dependence of FWHM on position and ROR in Figs. 4 and 5 is due to differences in speed of convergence. In experimental acquisitions, the number of iterations needs to be limited because of the decrease in signal-to-noise ratio as the number of iterations increases. This means a trade-off between resolution and acceptable noise level in the image must be established. ${ }^{6}$ As a result, when we limit the number of iterations, differences in resolution appear depending on the position of the point source in the FOV and on the ROR. The worst cases are found in the center of the FOV and for high ROR.

An accurate modeling of the system PSF is crucial to ensure the robustness of the iterative process and the avoidance of artifacts when a high number of iterations are performed. As Fig. 6 shows, the ability of the algorithm to separate images of hot rods placed in different regions of the sector phantom described above depends on the distance between the rods. For smaller distances between hot rods, the convergence speed was slower when the model was more accurate. Feng et al. ${ }^{16}$ 


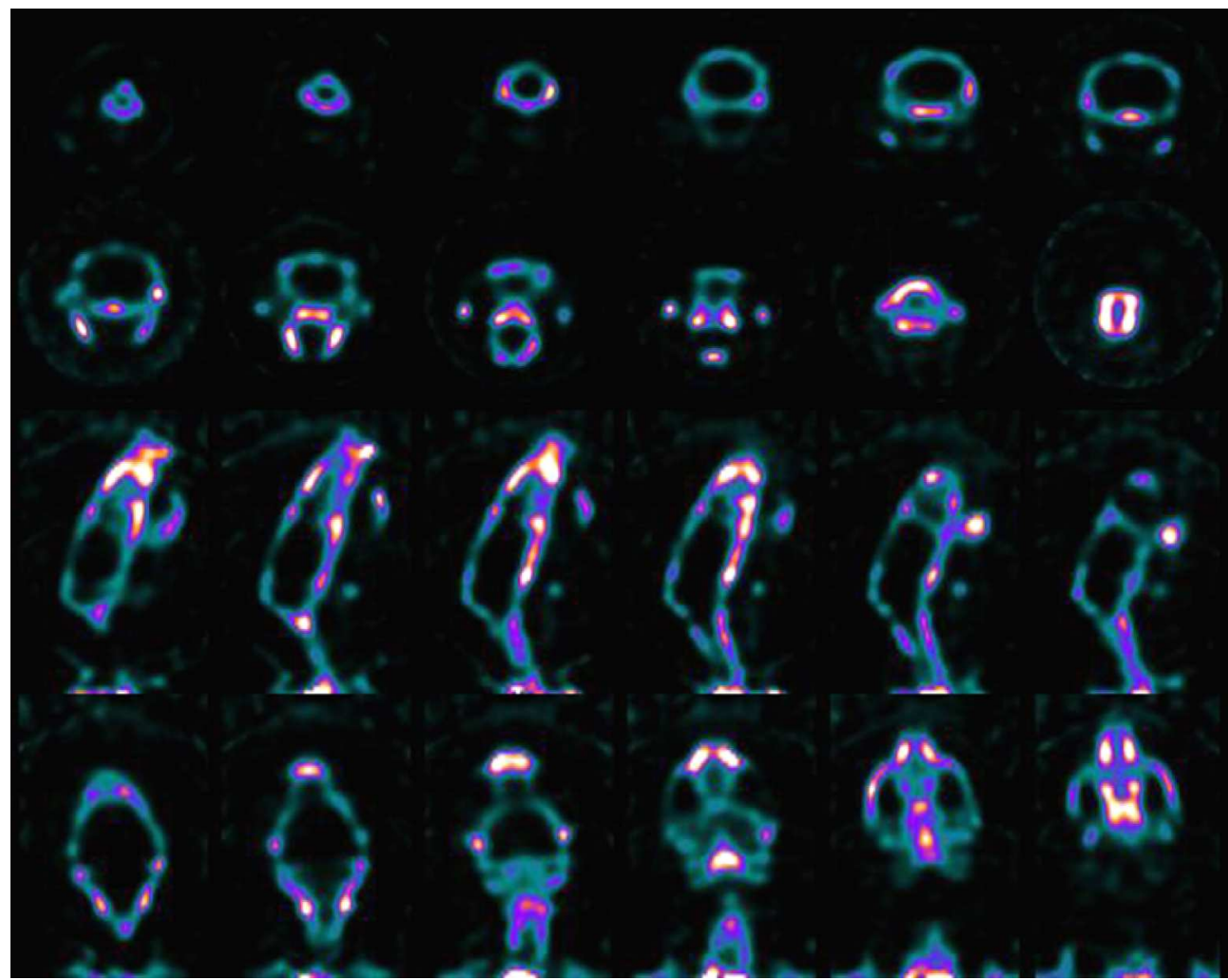

FIG. 13. Axial (a), sagittal (b), and coronal (c) slices of a ${ }^{99 m}$ Tc-HDP mouse head scan for SP-PSFi model for iteration 10.

also reported this effect, considering it to be related to the amount of information from a pixel that is projected onto the detector. Although convergence was slower when using more accurate models, resolution improved.

The second image-quality parameter studied was contrast, which was quantified using recovery coefficients and the percent contrast. As Fig. 7 shows, when corrections were incorporated in the PSF, the increase of noise with iterations was slower. As observed in Figs. 11(a)-11(e), for the same level of noise, better values of $\mathrm{RC}_{\mathrm{rod}}$ were obtained for all rod diameters when corrections were progressively incorporated in the PSF, although convergence was slower for more accurate models. It can be also observed in Figs. 11(a)-11(e) that with the exception of the $1 \mathrm{~mm}$ diameter rod, for a given rod diameter, differences between models of $\mathrm{RC}_{\text {rod }}$ were greater when the diameter was smaller. Thus, the improvement of $\mathrm{RC}_{\mathrm{rod}}$ using more accurate PSF models was greater for small rod diameters, with the inclusion of PSFi having a major impact.

For iterations higher than 12, a ring-type artifact appears in the image for all models, being most visible in the SPPSFi model (see Fig. 8). These artifacts are due to Gibbs phenomenon $^{28,29}$ and are observed for rod diameters greater than $4 \mathrm{~mm}$. As these artifacts are unacceptable for image visualization, it is necessary to stop the reconstruction process before they appear. As Fig. 9 shows, the ring-type artifact cannot be seen in SP-PSFi model until iteration 12. At this iteration, $\mathrm{RC}_{\text {rod }}$ and CNR (at iteration 10) values are clearly better for SP-PSFi model for the same level of noise. Stute and Comtat $^{32}$ proposed a method to suppress the ring-type artifact for PET imaging, performing an image-based PSF reconstruction and smoothing the estimate using the PSF as the convolution kernel. In our case, the final image obtained by smoothing the image reconstructed using SP-PSFi model would be similar to that obtained using SP model. As a consequence, the improvement of the inclusion of intrinsic PSF would be lost. In order to benefit from the inclusion of intrinsic PSF, whilst avoiding ring-type artifacts, it is necessary to study each system and limit the number of iterations used.

The percent contrast $Q_{H}$ of the hot cylinder improved with iterations for all PSF models. As Fig. 12 shows, $Q_{H}$ reached a plateau after a few iterations when $G$ model was used, thereby indicating a rapid convergence of the algorithm when only correction for the geometrical pinhole aperture is included in the transition matrix. Figure 12 also shows that $Q_{H}$ values of $82 \%, 82 \%$, and $86 \%$ were found when G, SP, or SP-PSFi models were used. These differences in $Q_{H}$ are lower than those observed for smaller diameter of rods in Figs. 11(a)-11(e). These findings suggest that an accurate PSF modeling is not as important for large objects as for smaller ones. Similar results were obtained for $Q_{C}$ in the cold cylinder, in agreement with other findings described in the literature for SPECT (Ref. 12) and PET (Ref. 30) when no scatter correction is performed.

\section{CONCLUSIONS}

We developed a reconstruction algorithm focused on a low magnification SPECT system equipped with a pinhole 
collimator. The algorithm incorporated PSFi response as a function of the incidence beam angle and the attenuation of gamma rays through the pinhole collimator into the transition matrix.

The influence of the inclusion of this PSFi on the values of several reconstructed image-quality parameters was assessed. The results derived from this evaluation demonstrate that PSFi plays a major role in the improvement of resolution, contrast, and recovery coefficients. Our findings also show that in order to avoid the appearance of ring-type artifacts, the number of iterations should be limited.

\section{ACKNOWLEDGMENTS}

This work was supported in part by The Spanish Ministry of Science \& Innovation (SAF2009-08076), CDTI-CENIT (AMIT project), and Fondo de Investigaciones Sanitarias (PI12-00390).

\footnotetext{
a) Author to whom correspondence should be addressed. Electronic mail: pablo.aguiar.fernandez@sergas.es; Telephone: 0034932607805.

${ }^{1}$ B. L. Franc, P. D. Acton, C. Mari, and B. H. Hasegawa, "Small-animal SPECT and SPECT/CT: Important tools for preclinical investigation," J. Nucl. Med. 49, 1651-1663 (2008).

${ }^{2}$ R. Weissleder, "Scaling down imaging: Molecular mapping of cancer in mice," Nat. Rev. Cancer 2, 11-18 (2002).

${ }^{3}$ F. Van der Have, B. Vastenhouw, R. M. Ramakers, W. Branderhorst, J. O. Krah, C. Ji, S. G. Staelens, and F. J. Beekman, "U-SPECT-II: An ultra-highresolution device for molecular small-animal imaging," J. Nucl. Med. 50, 599-605 (2009).

${ }^{4}$ N. Schramm, J. Hoppin, C. Lackas, F. Forrer, R. Valkema, and M. De Jong, "The NanoSPECT: A high-sensitivity multi-pinhole SPECT system with submillimeter (nanoliter) spatial resolution for imaging small rodents," J. Nucl. Med. 47(Suppl. 1), 233P (2006).

${ }^{5}$ F. P. Difilippo, "Design and performance of a multi-pinhole collimation device for small animal imaging with clinical SPECT and SPECT-CT scanners,” Phys. Med. Biol. 53, 4185-4201 (2008).

${ }^{6}$ S. D. Metzler, S. Vemulapalli, R. J. Jaszczak, G. Akabani, and B. B. Chin, "Feasibility of whole-body functional mouse imaging using helical pinhole SPECT,” Mol. Imaging Biol. 12, 35-41 (2009).

${ }^{7}$ L. R. Macdonald, B. E. Patt, J. S. Iwanczyk, S. Member, B. M. W. Tsui, Y. Wang, E. C. Frey, D. E. Wessell, P. D. Acton, and H. F. Kung, "Pinhole SPECT of mice using the LumaGEM gamma camera," IEEE Trans. Nucl. Sci. 48, 830-836 (2001).

${ }^{8}$ J. Y. Hesterman, M. A. Kupinski, L. R. Furenlid, D. W. Wilson, and H. H Barrett, "The multi-module, multi-resolution system (M[sup 3]R): A novel small-animal SPECT system,” Med. Phys. 34, 987-993 (2007).

${ }^{9}$ M. Freed, M. A. Kupinski, L. R. Furenlid, D. W. Wilson, and H. H. Barrett, "A prototype instrument for single pinhole small animal adaptive SPECT imaging," Med. Phys. 35, 1912-1925 (2008).

${ }^{10}$ K. Ogawa, N. Ohmura, H. Iida, K. Nakamura, T. Nakahara, and A. Kubo, "Development of an ultra-high resolution SPECT system with a CdTe semiconductor detector," Ann. Nucl. Med. 23, 763-770 (2009).

${ }^{11}$ C. Vanhove, M. Defrise, A. Bossuyt, and T. Lahoutte, "Improved quantification in single-pinhole and multiple-pinhole SPECT using micro-CT information,’ Eur. J. Nucl. Med. Mol. Imaging 36, 1049-1063 (2009).
}

${ }^{12}$ A. A. Harteveld, A. P. W. Meeuwis, J. A. Disselhorst, C. H. Slump, W. J. G. Oyen, O. C. Boerman, and E. P. Visser, "Using the NEMA NU 4 PET image quality phantom in multipinhole small-animal SPECT,” J. Nucl. Med. 52, 1646-1653 (2011).

${ }^{13}$ C. Wu, J. R. De Jong, H. A. Gratama van Ande, F. Van der have, B. Vastenhouw, P. Laverman, O. C. Boerman, R. A. Dierckx, and F. J. Beekman, "Quantitative multi-pinhole small-animal SPECT: Uniform versus nonuniform Chang attenuation correction,” Phys. Med. Biol. 56, N183-N193 (2011).

${ }^{14}$ C. Vanhove, M. Defrise, A. Bossuyt, and T. Lahoutte, "Improved quantification in multiple-pinhole SPECT by anatomy-based reconstruction using microCT information," Eur. J. Nucl. Med. Mol. Imaging 38, 153-165 (2011).

${ }^{15}$ C. Vanhove, A. Andreyev, M. Defrise, J. Nuyts, and A. Bossuyt, "Resolution recovery in pinhole SPECT based on multi-ray projections: A phantom study," Eur. J. Nucl. Med. Mol. Imaging 34, 170-180 (2007).

${ }^{16}$ B. Feng, M. Chen, B. Bai, A. M. Smith, D. W. Austin, R. A. Mintzer, D. Osborne, and J. Gregor, "Modeling of the point spread function by numerical calculations in single-pinhole and multipinhole SPECT reconstruction," IEEE Trans. Nucl. Sci. 57, 173-180 (2010).

${ }^{17}$ R. Accorsi and S. D. Metzler, "Analytic determination of the resolutionequivalent effective diameter of a pinhole collimator," IEEE Trans. Med. Imaging 23, 750-763 (2004).

${ }^{18}$ S. D. Metzler and R. Accorsi, "Resolution- versus sensitivity-effective diameter in pinhole collimation: Experimental verification,” Phys. Med. Biol. 50, 5005-5017 (2005).

${ }^{19}$ B. J. Mallard and M. J. Myers, "The performance of a gamma camera for the visualization of radioactive isotopes in vivo," Phys. Med. Biol. 8, 165-182 (1963).

${ }^{20}$ M. F. Smith and R. J. Jaszczak, "The effect of gamma ray penetration on angle-dependent sensitivity for pinhole collimation in nuclear medicine," Med. Phys. 24, 1701-1709 (1997).

${ }^{21}$ D. A. Weber and M. Ivanovic, "Pinhole SPECT: Ultra-high resolution imaging for small animal studies," J. Nucl. Med. 36, 2287-2289 (1995).

${ }^{22}$ F. Van der Have and F. J. Beekman, "Photon penetration and scatter in micro-pinhole imaging: A Monte Carlo investigation,” Phys. Med. Biol. 49, 1369-1386 (2004).

${ }^{23}$ F. Pino, N. Roé, A. Orero, C. Falcón, S. Rojas, J. M. Benlloch, D. Ros, and J. Pavía, "Development of a variable-radius pinhole SPECT system with a portable gamma camera," Rev. Esp. Med. Nucl. 30, 286-291 (2011).

${ }^{24}$ D. Bequé, J. Nuyts, G. Bormans, P. Suetens, and P. Dupont, "Characterization of pinhole SPECT acquisition geometry," IEEE Trans. Med. Imaging 22, 599-612 (2003).

${ }^{25}$ F. Sánchez, M. M. Fernández, M. Giménez, J. M. Benlloch, M. J. RodríguezAlvarez, F. García de Quirós, Ch. Lerche, N. Pavón, J. A. Palazón, J. Martínez, and A. Sebastiá, "Performance tests of two portable mini gamma cameras for medical applications," Med. Phys. 33, 4210-4220 (2006).

${ }^{26}$ A. Sánchez-Crespo and S. A. Larsson, "The influence of photon depth of interaction and non-collinear spread of annihilation photons on PET image spatial resolution,” Eur. J. Nucl. Med. Mol. Imaging 33, 940-947 (2006).

${ }^{27}$ NEMA Standards Publication NU 4-2008.

${ }^{28}$ D. L. Snyder, M. I. Miller, L. J. Thomas, and D. G. Politte, "Noise and edge artifacts in maximum-likelihood reconstructions for emission tomography," IEEE Trans. Med. Imaging 6, 228-238 (1987).

${ }^{29}$ R. Boellaard, "Need for standardization of 18F-FDG PET/CT for treatment response assessments," J. Nucl. Med. 52(Suppl. 2), 93S-100S (2011).

${ }^{30}$ R. Prasad, O. Ratib, and H. Zaidi, "NEMA NU-04-based performance characteristics of the LabPET-8TM small animal PET scanner," Phys. Med. Biol. 56, 6649-6664 (2011).

${ }^{31}$ S. C. Kappadath, "Effects of voxel size and iterative reconstruction parameters on the spatial resolution of ${ }^{99 m}$ Tc SPECT/CT," J. Appl. Clin. Med. Phys. 12, 210-220 (2011).

${ }^{32} \mathrm{~S}$. Stute and C. Comtat, "Practical considerations for image-based PSF and blobs reconstruction in PET,” Phys. Med. Biol. 58, 3849-3870 (2013). 